\title{
A Resistance Emulation Approach to Optimize the Wave Energy Harvesting for a Direct Drive Point Absorber
}

\author{
Hugo Mendonça and Sergio Martinez
}

\begin{abstract}
In general, a major challenge for the exploitation of renewable energies is to improve their efficiency. In electricity generation from the energy of ocean waves, not unlike other technologies, the converter must be optimized to make the energy harvesting economically feasible. This paper proposes a passive tuning control strategy of a point absorber in which the power captured is maximized by controlling the electromagnetic force of the generator with a resistance emulation approach. The proposed strategy consists of mapping the optimal values for regular waves and applying them to irregular waves. This strategy is tested in a wave energy converter in which the generator is connected to a boost rectifier converter whose controller is designed to emulate a resistance. The power electronics system implemented is validated by comparing its performance with the case in which the generator is directly connected to a resistive load. The simulation results show the effectiveness of the proposed strategy as the maximum captured power is concentrated around the optimal values previously calculated and with the same behavior for both excitations.
\end{abstract}

Index Terms-Boost rectifier, maximum captured power, point absorber, resistance emulation, wave energy converter (WEC).

\section{INTRODUCTION}

$\mathbf{T}$ HERE is a global consensus on the use of renewable energies. In this context, the sea has great energy potential to be exploited in different ways, and it has been the subject of many studies. A significant group of technologies is based on the conversion of the ocean wave energy into electricity by a wave energy converter (WEC). Several WEC technologies have been proposed, but they are still at research and development stage [1]-[3]. This work is focused on the point absorber converter developed at Uppsala University [4]. It is a direct drive WEC that is composed of a buoy connected to a power take-off (PTO) system on the seabed. The energy conversion in the PTO is made through a linear permanent magnet synchronous generator (LPMSG), a complex and somewhat expensive machine.
However, by using it, there is no need to apply any intermediate system, such as hydraulic or pneumatic, to convert the low speed from the ocean waves (frequency around $0.1 \mathrm{~Hz}$ ) into high speed for conventional rotary generators. In addition, not having such mechanical connections is very interesting from the point of view of reducing maintenance costs.

One method of optimizing, the wave energy harvesting is to define a buoy with appropriate geometry [5]. In addition, an important issue to be considered for the feasibility of harnessing wave energy is the control strategy upon the WEC, on which both design and operation are highly dependent [6]. In this sense, [7] assesses how the geometry optimization works with usual control methods. The controller must be designed to deal with the natural irregularity of oceanic waves in amplitude, phase, and direction [8]. There are many control strategies that could be applied depending on the specific characteristics of each WEC [6], [9]. In the case of heaving buoys, one of the most widespread strategies is passive tuning, based on the application of a resisting force proportional to velocity [10], where the ratio of force to velocity is called the damping coefficient. In this type of tuning, the sea state is taken into account to calculate the optimal damping coefficient. On the other hand, active tuning is based on estimating the dominant wave frequency by an analysis of measured wave elevation and device velocity [11]. The fact that the incoming wave has to be known brings some difficulty to this method. Regarding this issue, prediction of variables such as force and velocity in a time horizon is required, as can be seen in [12] and [13]. Based on these predictions, different methods to optimize the captured power can be found in [13]-[15]. Furthermore, it is important to ensure an efficient control of a single WEC so that the strategy can be extended to the control of a wave farm, as in [16].

A WEC with an LPMSG requires power electronics to convert the generated electrical energy to a form suitable for the external electrical grid. The contribution of this work is an implementation of the control strategy of the electronic converter to improve the efficiency of the given WEC. The scheme, within the passive tuning approach, is to optimize the energy harvesting from the waves by varying the damping coefficient that, in turn, is modified by acting on the value of the equivalent resistance seen by the electrical generator. For this purpose, the ac-dc stage of the converter is implemented with a boost rectifier circuit to reach a unitary power factor, i.e., to emulate a resistive load as seen by the generator terminals.

In this paper, Section II describes the WEC and PTO models, and Section III details the proposed control strategy as well as 


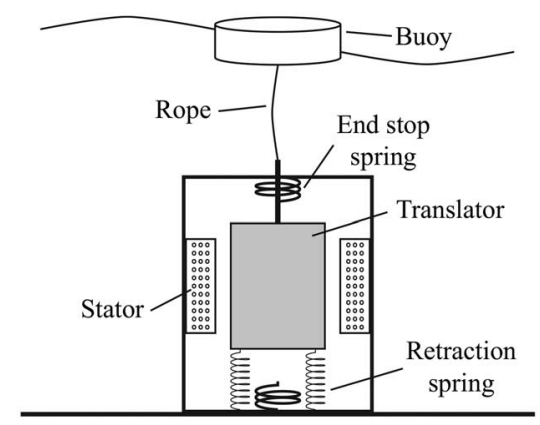

Fig. 1. Schematic representation of the WEC.

its implementation. Finally, the behavior of the whole system is assessed under different conditions, including generated and real wave data, and the most significant results are presented in Section IV.

\section{WEC DYNAMIC MODEL}

In this work, the one degree of freedom point absorber from [4], which is schematically shown in Fig. 1, has been implemented in SIMULINK/MATLAB, where the input to the model is the incoming wave. Relevant specifications are listed in Table I. A cylindrical buoy is connected to the translator by a rope with high stiffness. The translator is the moving part of the LPMSG, where the magnets are set, and the stator is the static part with the windings. The nominal values of this generator related to the nominal speed of the translator $\left(\nu_{n}\right)$ are also given in Table I. In order to avoid damage on the PTO structure during the translator motion, there are upper and lower end stop springs to limit the maximum stroke. In addition, another spring connects the translator to the bottom of the structure. This spring applies a downward force on the the translator during wave troughs and an initial force $F_{0}$ to reach a specific buoy draft when the system is in equilibrium. The characteristics of the WEC are based on the compilation of information available for the converter L1 [17]-[20]. Finally, the LPMSG is connected to a power electronic converter.

\section{A. Equation of Motion}

The equation of motion is based on the linear theory, which takes into account that the fluid is incompressible (constant density), frictionless, and irrotational. Also, the wave amplitudes are considered small compared to the wave length [21], [22]. This is a reasonable approximation in the most frequent sea states, where the motion is limited. A discussion on modeling higher order hydrodynamic effects can be found in [6, pp. 37-38] and references therein. As previously stated, the system under study is a circular cylinder buoy partially submerged, in a heave motion, according to the model presented in [17]. The system consists of the following coupled integrodifferential equations in which $x$ is the translator position and $y$ is the buoy position

$$
\begin{aligned}
& \left(m_{b}+m_{b}^{\infty}\right) \ddot{y}(t)+\int_{-\infty}^{t} k_{r}(t-\tau) \dot{y}(\tau) \mathrm{d} \tau+\rho g S_{w} y(t) \\
& \quad=F_{e}(t)-F_{w}(t)
\end{aligned}
$$

$$
m_{t} \ddot{x}(t)=F_{w}(t)-W_{t}-F_{\text {em }}(t)-F_{s}(t)+F_{\text {stop }}(t) .
$$

Equation (1a) related to the buoy is derived from the Cummins equation [23] where $y, \dot{y}, \ddot{y}, m_{b}, m_{b}^{\infty}, k_{r}, S_{w}, F_{e}$, and $F_{w}$ stand for: vertical displacement, speed, acceleration, inertial mass, added mass at high frequencies, radiation impulse response function (IRF), water plane area, excitation force, and line force. Equation (1b) is related to the translator motion, where $x, \ddot{x}, m_{t}, W_{t}$ are the displacement, acceleration, mass, and weight force of the translator, and $F_{\mathrm{em}}, F_{s}$, and $F_{\text {stop }}$ are the electromagnetic, retraction, and end stop forces.

The line force $F_{w}$ on the connection between the buoy and the translator acts as a coupling point between both systems, buoy and electrical generator. Depending on the displacement of the buoy and the translator, $F_{w}$ can be null, decoupling both systems, as described by the model

$$
F_{w}= \begin{cases}k_{w}(y-x), & \text { if } y>x \\ 0, & \text { else }\end{cases}
$$

where $k_{w}$ is the rope stiffness.

Added mass at high frequencies and the radiation IRF are calculated from the hydrodynamic parameters of the buoy, which are given in the frequency domain. These hydrodynamic parameters can be derived numerically, e.g., using software packages such as ANSYS Aqwa [24], the one used in this work. The hydrodynamic parameters are: added mass $A\left(\omega_{w}\right)$, potential damping $B\left(\omega_{w}\right)$, and the Froude-Kriloff and diffraction forces, also known as wave-to-force response amplitude operator $(\mathrm{RAO}) \tau_{\mathrm{RAO}}$, where $w_{w}$ is the wave angular frequency [25]. $m_{b}^{\infty}$ is obtained from the buoy added mass at high frequencies $A(\infty)$. The radiation IRF $k_{r}$ according to [26] can be calculated by

$$
k_{r}(t)=\frac{2}{\pi} \int_{0}^{\infty} B\left(\omega_{w}\right) \cos \left(\omega_{w} t\right) \mathrm{d} \omega_{w} .
$$

In this work, $k_{r}$ is represented by a state space model using the method proposed in [26]. Other identification methods for similar application can be found in [27]-[29]. The excitation force can be defined as follows:

$$
F_{e}(t)=\int_{-\infty}^{+\infty} k_{e}(t-\tau) \zeta(\tau) \mathrm{d} \tau
$$

where $\zeta(t)$ is the elevation of the sea surface and $k_{e}(t)$ is the wave excitation IRF calculated by

$$
k_{e}(t)=\frac{1}{2 \pi} \int_{-\infty}^{+\infty} K_{e}\left(j \omega_{w}\right) e^{j \omega_{w} t} \mathrm{~d} \omega_{w} .
$$

Equation (5) is the inverse Fourier transform of the complex frequency response function $K_{e}\left(j \omega_{w}\right)$ that may be given in terms of the wave-to-force RAO

$$
K_{e}\left(j \omega_{w}\right)=\left|\tau_{\mathrm{RAO}}\left(\omega_{w}\right)\right| \cdot e^{j \phi_{\tau_{\mathrm{RAO}}}\left(\omega_{w}\right)}
$$

where $\left|\tau_{\mathrm{RAO}}\left(\omega_{w}\right)\right|$ and $\phi_{\tau_{\mathrm{RAO}}}\left(\omega_{w}\right)$ are the amplitude and phase of the wave-to-force RAO, respectively. The identification method applied to the wave excitation IRF is the same as the 
TABLE I

Main Electrical and Mechanical Parameters of WeC

\begin{tabular}{crcrcr}
\hline \multicolumn{1}{c}{ Buoy } & \multicolumn{2}{c}{ Linear generator } & \multicolumn{2}{c}{ Mechanical system } \\
\hline Diameter & $3 \mathrm{~m}$ & Nominal speed $\nu_{n}$ & $0.7 \mathrm{~m} / \mathrm{s}$ & Upper spring length $l_{u}$ & $250 \mathrm{~mm}$ \\
Height & $0.8 \mathrm{~m}$ & Line to line nominal RMS voltage $V_{n}$ & $200 \mathrm{~V}$ & Lower spring length $l_{l}$ & $140 \mathrm{~mm}$ \\
Mass & $1000 \mathrm{~kg}$ & Nominal Power $P_{n}$ & $10 \mathrm{~kW}$ & Upper stiffness $k_{u}$ & $243 \mathrm{kN} / \mathrm{m}$ \\
Draft at still water level & $0.4 \mathrm{~m}$ & Pole width $\tau_{p}$ & $50 \mathrm{~mm}$ & Lower stiffness $k_{l}$ & $215 \mathrm{kN} / \mathrm{m}$ \\
& & Synchronous inductance $L_{s}$ & $11.7 \mathrm{mH}$ & Energy storage stiffness $k_{s}$ & $6.20 \mathrm{kN} / \mathrm{m}$ \\
& & Armature resistance $R_{s}$ & $0.44 \Omega$ & Rope stiffness $k_{w}$ & $450 \mathrm{kN} / \mathrm{m}$ \\
& & Translator weight & $1000 \mathrm{~kg}$ & Initial spring force $F_{0}$ & $8.12 \mathrm{kN}$ \\
\hline
\end{tabular}

one applied to the radiation IRF. However, the wave excitation IRF is noncausal which results in an unstable representation either by a transfer function or by state space variables. One way to avoid this problem is to make the wave excitation IRF "approximately causal" as presented in [28].

$F_{\text {em }}$ is the electromagnetic force provided by the linear generator detailed in Section II-B. The retraction force $F_{s}$ is the force applied by the spring at the bottom of the structure and modeled by

$$
F_{s}=F_{0}+k_{s} x
$$

where $k_{s}$ is the spring stiffness, and $F_{0}$ is the force of the retraction spring in the equilibrium. The end stop force $F_{\text {stop }}$ is the force applied by the upper and lower springs to break the translator motion. $F_{\text {stop }}$ is decomposed into

$$
\begin{aligned}
& F_{u}= \begin{cases}k_{u}\left(x-l_{u}\right), & \text { if } l_{u}<x \\
0, & \text { else }\end{cases} \\
& F_{l}= \begin{cases}-k_{l}\left(l_{l}+x\right), & \text { if } l_{l}<-x \\
0, & \text { else }\end{cases}
\end{aligned}
$$

where $F_{u}$ and $F_{l}$ are the upper and lower end forces, $k_{u}$ and $k_{l}$ are the the upper and lower spring stiffness, and $l_{u}$ and $l_{l}$ are the upper and lower spring lengths.

\section{B. Linear Permanent Magnet Synchronous Generator}

The dynamic model of this machine is similar to a rotating synchronous generator. In a linear generator, the mechanical translation is in synchronism with the magnetic field. As in a rotating generator, it is common to express it by a rotating reference frame that moves at synchronous speed $\omega$ ( $d q$-model) [30]. If $\boldsymbol{v}_{a b c}=\left[\begin{array}{lll}v_{a} & v_{b} & v_{c}\end{array}\right]^{T}$ is a generic three-phase vector, the $d q 0$ variables are calculated by $\left[v_{d} v_{q} v_{0}\right]^{T}=\mathbf{K} \cdot \boldsymbol{v}_{a b c}$, with

$$
\mathbf{K}=\frac{2}{3}\left[\begin{array}{ccc}
\cos \theta & \cos \left(\theta-\frac{2 \pi}{3}\right) & \cos \left(\theta+\frac{2 \pi}{3}\right) \\
-\sin \theta & -\sin \left(\theta-\frac{2 \pi}{3}\right) & -\sin \left(\theta+\frac{2 \pi}{3}\right) \\
\frac{1}{2} & \frac{1}{2} & \frac{1}{2}
\end{array}\right]
$$

where $\theta=\omega t$. As the LPMSG is balanced, then $v_{0}=0$. The linear speed of the translator is related to the electrical angular frequency by

$$
\dot{x}=2 f \tau_{p}=\frac{\omega}{\pi} \tau_{p}
$$

where $\dot{x}, f$, and $\tau_{p}$ are translator speed, electrical frequency, and pole width, respectively. The $d q$-model equations for the LPMSG are as follows:

$$
\begin{aligned}
& e_{d}=-R_{s} i_{d}+\frac{\mathrm{d} \lambda_{d}}{\mathrm{~d} t}-\omega \lambda_{q} \\
& e_{q}=-R_{s} i_{q}+\frac{\mathrm{d} \lambda_{q}}{\mathrm{~d} t}+\omega \lambda_{d}
\end{aligned}
$$

where $e_{d}, e_{q}$ and $i_{d}, i_{q}$ are the back EMF voltages and currents in the direct and quadrature axes, $R_{s}$ is the armature resistance, and $\lambda_{d}, \lambda_{q}$ are the linkage flux described by

$$
\lambda_{d}=-L_{s} i_{d}+\lambda_{\mathrm{PM}} ; \lambda_{q}=-L_{s} i_{q}
$$

where $L_{s}$ is the synchronous inductance and $\lambda_{\mathrm{PM}}$ is the total flux from the permanent magnet (PM).

The three-phase power output of the stator in $d q$-model is

$$
P_{\text {gen }}=\frac{3}{2} \cdot\left(e_{d} i_{d}+e_{q} i_{q}\right) .
$$

From (13), the electromagnetic power, i.e., the power transferred across the air-gap, is

$$
P_{\mathrm{em}}=\frac{3}{2} \cdot \omega\left(\lambda_{d} i_{q}-\lambda_{q} i_{d}\right) .
$$

The electromagnetic force that opposes the motion as a damping force is

$$
F_{\mathrm{em}}=\frac{P_{\mathrm{em}}}{\dot{x}}=\frac{3}{2} \cdot \frac{\pi}{\tau_{p}}\left(\lambda_{d} i_{q}-\lambda_{q} i_{d}\right) .
$$

Finally, the active area of the stator, i.e., the area of the stator covered by the translator, is assumed to be constant and not dependent on the translator position.

\section{Control Strategy}

Some preliminary results about the behavior of the model described in Section II can be found in [31], where the WEC is connected directly to a three-phase resistive load (Fig. 2) with no control action. In general, the control system of a point absorber oscillating in heave actuates through the PTO, and it is based on the fact that the position of the actuator and the buoy are directly related. One particularity of the WEC of this work is that there are some situations in which this is not true, particularly when $y \leq x$, resulting in independent dynamics between 


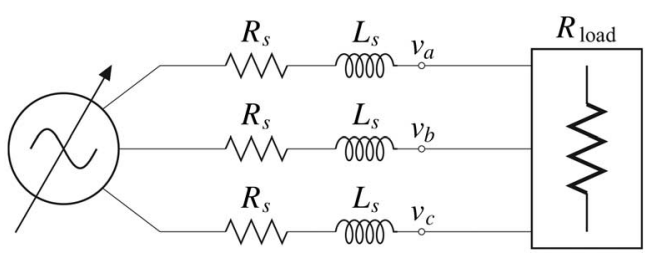

Fig. 2. WEC connected to three-phase resistive load.

buoy and translator. With a similar approach as in [32], in the sense of using a sea state based optimization, this section proposes a control strategy to maximize the power extraction for each given sea state and its practical implementation through a variable reactive force in the linear generator.

\section{A. Optimal Power Point}

At this point, regular waves, i.e., harmonic waves, as described in [33], are used to excite the system. Their waveenergy transport is defined by

$$
J=\frac{\rho g^{2}}{32 \pi} T H^{2}
$$

given in (W/m), where $\rho, g, T$, and $H$ are fluid density, acceleration due to gravity, and wave period and height. It is important to notice that $H$ is twice the wave amplitude.

The electromagnetic force developed by the linear generator can be modeled as a viscous damping, which leads to a damping coefficient proportional to the translator speed described by $F_{\mathrm{em}}=\gamma \cdot \dot{x}$ [34]. As the electromagnetic force can be calculated by (15), the damping coefficient can be found for a given speed. In terms of power, the damping equation can be rewritten as $P_{\mathrm{em}}=\gamma \cdot \dot{x}^{2}$. Further, the incident power on the buoy is $P_{b}=J \cdot d_{b}$, where $d_{b}$ is the buoy diameter. Thus, the ratio of captured power can be defined as $P_{\text {ratio }}=P_{\mathrm{em}} / P_{b}$. However, the power captured in reality is hardly above $20 \%$ of the waveenergy transport [35] and many efforts have been devoted to optimize the captured power. For example, [14], [15], [36], and [37] present optimization methods for generic PTOs by acting directly on the damping coefficient. These methods are usually compared to established control strategies as latching [38] and declutching [39]. However, in this work, the comparison with a latching control is not possible because the buoy is not rigidly connected to the PTO, as generic ones are, and the system is unable to "lock" its motion, and the comparison with the declutching control is not feasible due to electrical instabilities in the converter when unloading the LPMSG.

In this work, the WEC is connected to a three-phase resistive load $R_{\text {load }}$ as shown in Fig. 2. The system has been subjected to multiple excitation where the period $(T)$ was varied between 2 and $16 \mathrm{~s}$ with steps of $0.25 \mathrm{~s}$, and the height $(H)$ was varied between 0.1 and $2.7 \mathrm{~m}$ with steps of $0.1 \mathrm{~m}$. These wide ranges of periods and heights were chosen not taking into account any specific place. For each period and height, the system was loaded with different values of resistance. It is observed that each load is related to a PTO damping coefficient, as shown in Fig. 3, where the higher the load, the lower the PTO damping coefficient. This relationship is independent of $H$ and $T$ and it

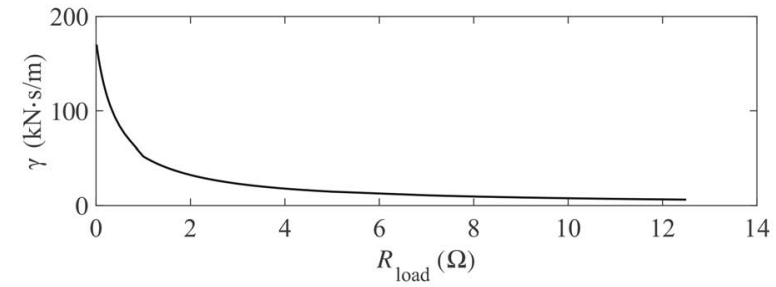

Fig. 3. Damping coefficient versus resistive load.

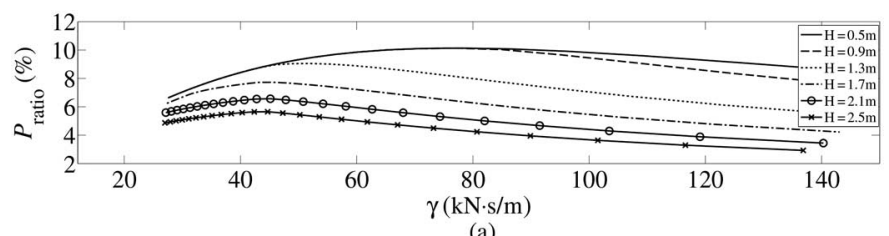

(a)

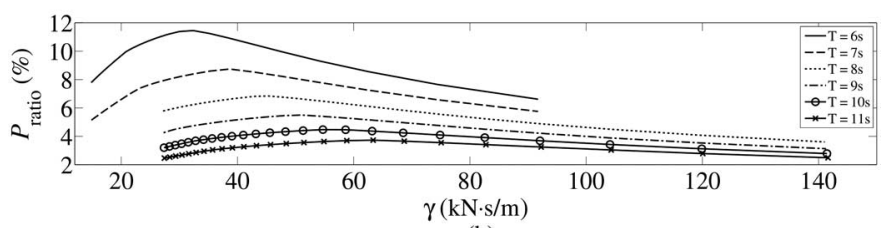

(b)

Fig. 4. Captured power ratio versus damping coefficient for regular waves. (a) Period is kept constant and the height varies (wave period, $T=8 \mathrm{~s}$ ). (b) Height is kept constant and the period varies (wave height, $H=2 \mathrm{~m}$ ).

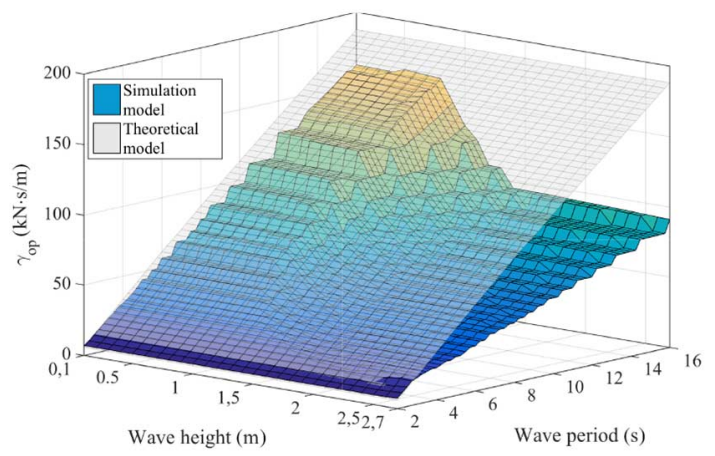

Fig. 5. Optimal damping coefficient $\gamma_{\text {op }}$ with respect to wave height and period for the simulation and theoretical models.

is not a generic curve, being highly dependent on the generator characteristics. This relationship is an important aspect for a direct drive WEC, as it is possible to take advantage of the power electronic converter connected to the LPMSG to control the PTO force, as it is explained in Section III-B.

These simulations also allow the evaluation of the captured power. Note that the captured power is the power developed by the LPMSG rather than the buoy. Fig. 4 shows the ratio of captured power profile ( $P_{\text {ratio }} \gamma$-curves) for some sets of period and height. For each $P_{\text {ratio }} \gamma$-curve, it is possible to identify the maximum power point and the respective PTO damping coefficient that is defined as the optimal damping coefficient $\gamma_{\text {op }}$, which, in turn, is associated with the optimal resistance $R_{\text {op }}$. Finally, the optimal damping coefficients can be organized with respect to period and height as shown in the colored surface of Fig. 5.

For comparison, the system has been modeled in a simple way with a linear electrical analogue as in [40], and taking 


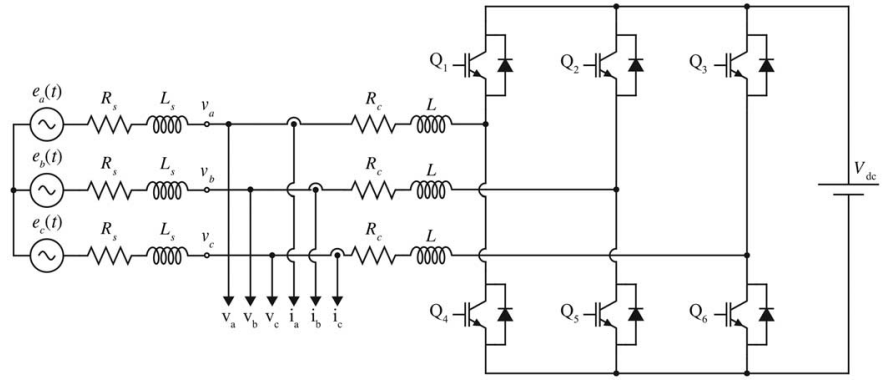

Fig. 6. WEC connected to the boost rectifier.

into account the following linearizing assumptions: the translator and the buoy are rigidly coupled $(y=x)$, and the action of the end stop springs is neglected $\left(F_{\text {stop }}=0\right)$. Thus, the optimal damping coefficients (shown in the gray surface of Fig. 5) can be theoretically calculated by applying the maximum power transfer theorem. In addition, it is verified in Fig. 5 that the more the nonlinearities are present in the system, the greater the difference between both results is.

Realistic seas do not have periodic waves. Usually, they are described by statistical parameters such as energy period and significant wave height. The approach of using the optimal values obtained from height and period of regular waves, instead of these statistical parameters, as a simplification for irregular waves, is applied in this work and it can be found in others also, e.g., [14], [41].

\section{B. Resistance Emulation}

Since the proposed method is based on that, for given values of wave height and period, there is a resistance related to the maximum power point, the objective is to implement a circuit able to emulate that resistance seen by the linear generator that allows the WEC to extract the desired power. Emulating a resistance means to make current proportional to voltage and, therefore, to reach unitary power factor. One way to obtain it is by using the three-phase ac-dc boost rectifier [42]. This converter, whose topology is shown in Fig. 6, is connected to the linear generator by a submarine cable. It is made up of six single-pole single-throw (SPST) current-bidirectional twoquadrant switches and requires an inductor in the ac-side to filter high-frequency harmonics. The dc-side is modeled by a dc-source. The boost rectifier operation requires the dc voltage $V_{\mathrm{dc}}$ to be greater than the peak value of the line-to-line input voltage. The switches of each phase are complementary, i.e., while $\mathrm{Q}_{1}$ is conducting, $\mathrm{Q}_{4}$ is blocked. The emulated resistance $R_{e}$ is obtained by controlling the switching. In other words, the switching sequence provides a voltage in the ac-side of the converter that makes voltage and current proportional at the measurement point.

The switching strategy implemented to emulate the resistor was the hysteresis-band current control [43], whose principle is shown in Fig. 7. The hysteresis control is based on a nonlinear feedback loop with two-level hysteresis comparator. The switching signals are produced when the absolute error $\left|i^{*}-i\right|$ exceeds the band $\beta / 2$. In this way, each measured current

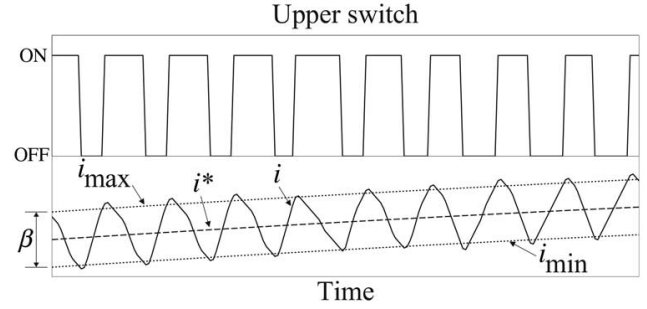

Fig. 7. Principle of hysteresis-band current control.

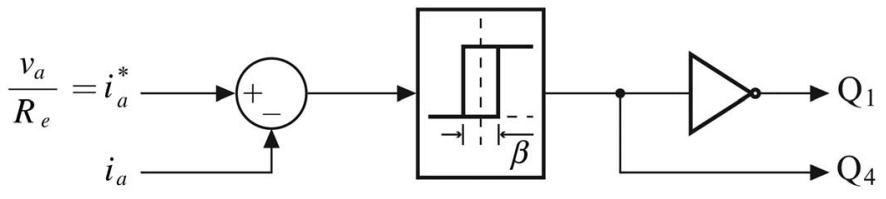

Fig. 8. Block diagram implemented for hysteresis control per phase.

$\left(i_{a}, i_{b}\right.$, or $\left.i_{c}\right)$ is guided to follow its reference $\left(i_{a}^{*}, i_{b}^{*}\right.$, or $\left.i_{c}^{*}\right)$ within the tolerance band $\beta$. One possibility of implementation is to use three independent controllers, one for each phase, as the one shown in Fig. 8 for phase $a$. In this case, the upper switch $Q_{1}$ turns on when the measured current $i_{a}$ is greater than the upper limit of the band $\left(i_{\max }\right)$, and it turns OFF when $i_{a}$ is lower than the lower limit $\left(i_{\min }\right)$. Note that the logic presented in Fig. 8 corresponds to the phase current polarity taken from the generator to the boost rectifier. The special advantage of this controller is the fact that there is no tracking errors (very fast response), which makes it ideally suited for this type of generation as the voltage generated is constantly varying according to the incoming wave. The current is supposed to be kept within the hysteresis band; however, if there is no neutral connection in the circuit, in the case of three independent controllers, the actual current can reach double value of the hysteresis bandwidth [43]. As the emulated resistance must be seen by the generator terminals, the phase voltage and the line current are measured at this point. A current reference is generated for each phase, and it is defined by the quotient between the phase voltage and the required resistance $R_{e}$ as shown in Fig. 8.

\section{Simulations And Results}

The proposed method to maximize the captured power is based on the adjustment of the damping coefficient of the electrical generator by setting an equivalent resistance seen by its terminals. The optimal damping map was obtained by exciting the system with regular waves for several combinations of wave period and height (Fig. 5), and a boost rectifier (described in Section III-B) was implemented to set the optimal resistance ( $\left.R_{\text {op }}\right)$ associated with the optimal damping. The simulations of this system are presented in this section and they are intended to illustrate the applicability of the implemented electronic circuit and to verify that the proposed control strategy achieves the maximum captured power.

Different time series of irregular waves have been generated according to [21] by using the ITTC spectrum, which is a variation of the two-parameter Pierson-Moskowitz spectrum [44]. In this work, the statistical parameters used are the energy 


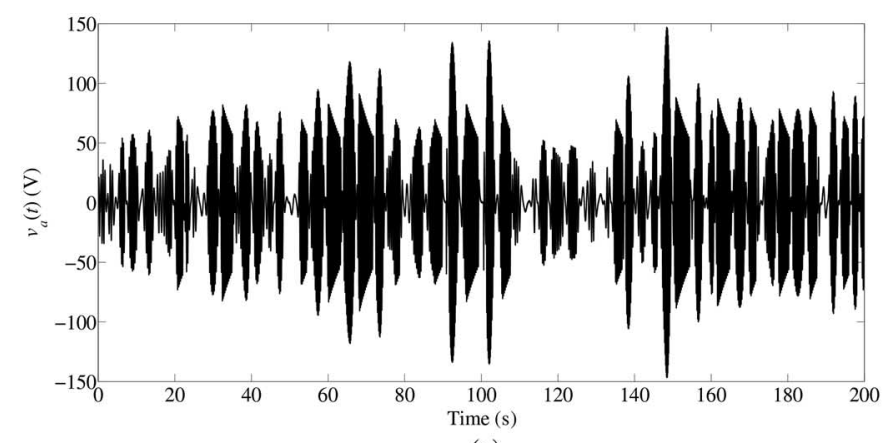

(a)

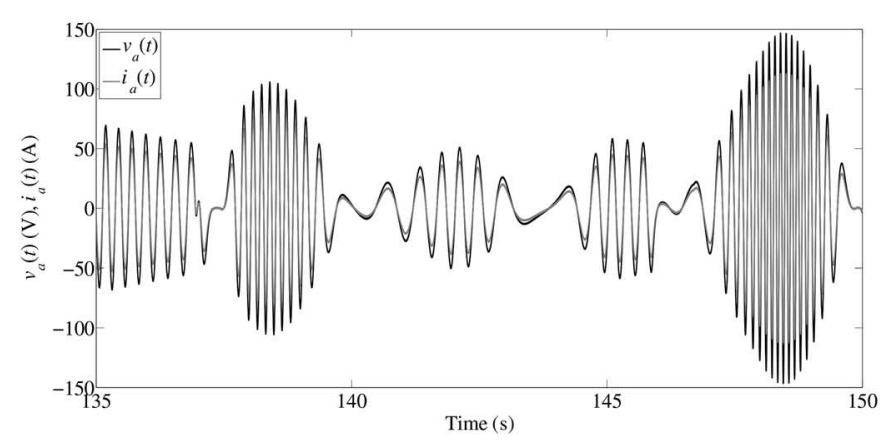

(b)

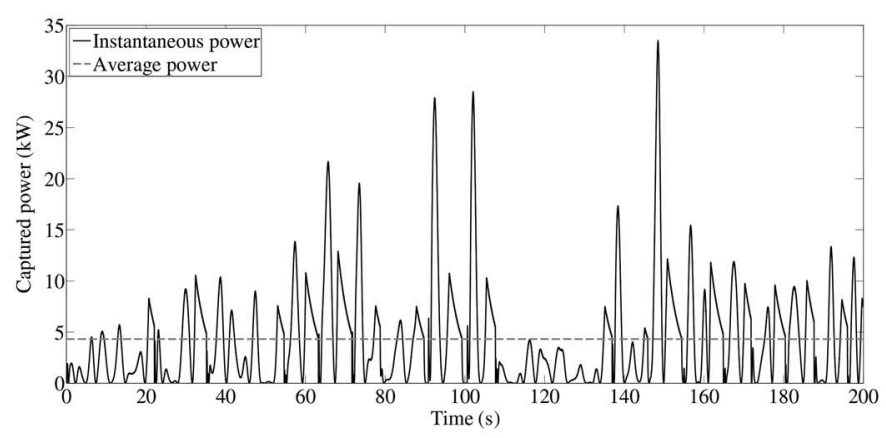

(c)

Fig. 9. Simulation of the WEC (Fig. 6) for an irregular wave with $T_{e}=8 \mathrm{~s}$ and $H_{s}=2 \mathrm{~m}$, and the boost rectifier controller set to $1.3 \Omega$. (a) Phase voltage, (b) phase voltage and line current, and (c) captured power for the WEC connected to the boost rectifier.

period $T_{e}$ and the significant wave height $H_{s}$. The wave-energy transport, in this case, is [1]

$$
J=\frac{\rho g^{2}}{64 \pi} T_{e} H_{s}^{2}
$$

\section{A. WEC Operation}

To assess the proper operation of the boost rectifier, the system illustrated in Fig. 6 was simulated with a wave time series generated with $T_{e}=8 \mathrm{~s}$ and $H_{s}=2 \mathrm{~m}$. For this specific wave state, according to Section III, the load seen by the generator terminals is set to $1.3 \Omega$, which, in turn, is associated with the optimal damping. The electrical generator is connected to the boost rectifier through a cable in series with a filter whose equivalent resistance and inductance are $R_{c}=0.44 \Omega$ and $L=$ $100 \mathrm{mH}$, respectively.

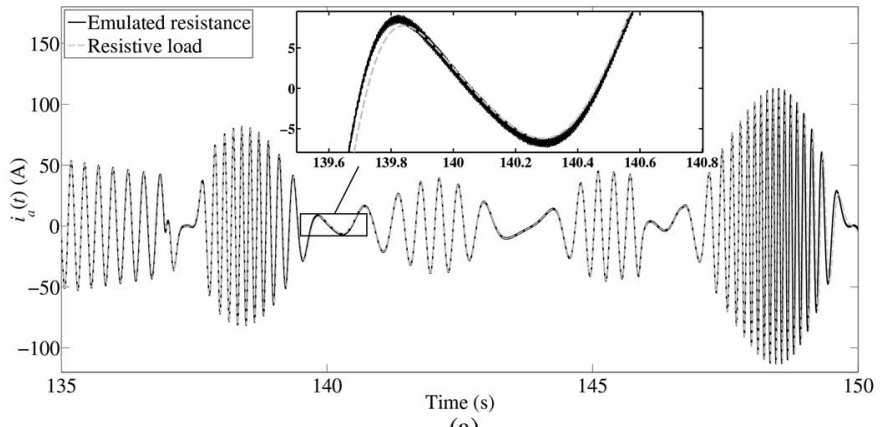

(a)

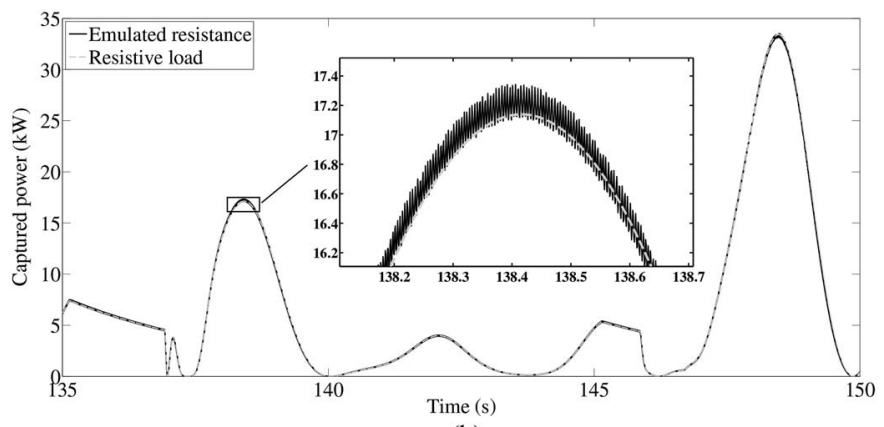

(b)

Fig. 10. Comparison between the system with emulated resistance and the ideal system when excited by an irregular wave with $T_{e}=8 \mathrm{~s}$ and $H_{s}=$ $2 \mathrm{~m}$. Resistive load and emulated resistance are $1.3 \Omega$. (a) Line currents and (b) captured power for both cases.

The main waveforms resulting from this simulations are presented in Fig. 9. The generated phase $a$ voltage $v_{a}(t)$ is presented in Fig. 9(a) for the first $200 \mathrm{~s}$ of simulation. Fig. 9(b) shows a time zoom of $v_{a}(t)$ along with the corresponding line current $i_{a}(t)$ illustrating that the implemented boost converter achieves its goal of keeping voltage and current in phase. The captured power profile is presented in Fig. 9(c), where the dashed line is the average power $\left(P_{\mathrm{av}}=4.32 \mathrm{~kW}\right)$.

To show the performance of the emulation resistance approach to control the damping coefficient and, thus, to achieve the maximum power point, the results obtained in the previous simulation are contrasted with the ideal case in Fig. 10. In the ideal case, the WEC is connected directly to a threephase resistive load (Fig. 2) and it is excited with the same wave time series. Fig. 10(a) shows the current in both models, and Fig. 10(b) shows the captured power. In both graphics, it is observed that the waveforms are practically the same. There is a very tiny phase shift between them because the measured voltage, used to calculate the current reference in Fig. 8, must be filtered at high frequencies. Another difference is the ripple due to switching in the boost rectifier. Although the system has a variable switching frequency, it is always below $10 \mathrm{kHz}$, which is a reasonable rate in electrical power systems, for a hysteresis band of $\pm 0.4 \mathrm{~A}$.

\section{B. Performance of the Proposed Control Strategy}

Another group of simulations was carried out to verify the effectiveness of the strategy proposed in this paper. For this purpose, sixteen different wave time series of irregular waves 
TABLE II

COMPARISON OF THE CAPTURED POWER FOR IRREgUlar WAVE Profiles With Different Values of Emulated Resistance

\begin{tabular}{|c|c|c|c|c|c|c|c|}
\hline \multirow{4}{*}{$T_{e}(\mathrm{~s})$} & \multirow{4}{*}{$H_{s}(\mathrm{~m})$} & \multirow{4}{*}{$\begin{array}{l}\text { Wave time } \\
\text { series }\end{array}$} & \multicolumn{5}{|c|}{ Emulated resistances $(\Omega)$} \\
\hline & & & $R_{1}$ & $R_{2}$ & $R_{\mathrm{op}}$ & $R_{3}$ & $R_{4}$ \\
\hline & & & \multicolumn{5}{|c|}{ Average captured power $(\mathrm{kW})$} \\
\hline & & & $P_{R_{1}}$ & $P_{R_{2}}$ & $P_{R_{\mathrm{op}}}$ & $P_{R_{3}}$ & $P_{R_{4}}$ \\
\hline \multirow{2}{*}{8} & \multirow{2}{*}{1} & \multirow{2}{*}{ Wave 1} & 0.25 & 0.60 & 0.70 & 0.80 & 1.50 \\
\hline & & & 0.69 & 0.80 & 0.81 & 0.81 & 0.76 \\
\hline \multirow{2}{*}{8} & \multirow{2}{*}{1} & \multirow{2}{*}{ Wave 2} & 0.25 & 0.60 & 0.70 & 0.80 & 1.50 \\
\hline & & & 1.02 & 1.17 & 1.18 & 1.19 & 1.11 \\
\hline \multirow{2}{*}{8} & \multirow{2}{*}{1} & \multirow{2}{*}{ Wave 3} & 0.25 & 0.60 & 0.70 & 0.80 & 1.50 \\
\hline & & & 0.87 & 1.02 & 1.04 & 1.05 & 0.98 \\
\hline \multirow{2}{*}{8} & \multirow{2}{*}{1} & \multirow{2}{*}{ Wave 4} & 0.25 & 0.60 & 0.70 & 0.80 & 1.50 \\
\hline & & & 0.71 & 0.83 & 0.84 & 0.85 & 0.80 \\
\hline \multirow{2}{*}{8} & \multirow{2}{*}{2} & \multirow{2}{*}{ Wave 5} & 0.50 & 1.20 & 1.30 & 1.40 & 2.00 \\
\hline & & & 3.37 & 3.88 & 3.88 & 3.86 & 3.63 \\
\hline \multirow{2}{*}{8} & \multirow{2}{*}{2} & \multirow{2}{*}{ Wave 6} & 0.50 & 1.20 & 1.30 & 1.40 & 2.00 \\
\hline & & & 3.77 & 4.32 & 4.32 & 4.30 & 4.00 \\
\hline \multirow{2}{*}{8} & \multirow{2}{*}{2} & \multirow{2}{*}{ Wave 7} & 0.50 & 1.20 & 1.30 & 1.40 & 2.00 \\
\hline & & & 3.41 & 3.97 & 3.94 & 3.91 & 3.54 \\
\hline \multirow{2}{*}{8} & & & 0.50 & 1.20 & 1.30 & 1.40 & 2.00 \\
\hline & 2 & Wave 8 & 3.61 & 4.23 & 4.23 & 4.21 & 3.89 \\
\hline 15 & 1 & Weye 0 & 0.05 & 0.10 & 0.20 & 0.30 & 1.00 \\
\hline 15 & 1 & Wave 9 & 0.49 & 0.50 & 0.53 & 0.53 & 0.46 \\
\hline 15 & 1 & Waye 10 & 0.05 & 0.10 & 0.20 & 0.30 & 1.00 \\
\hline 15 & 1 & Wave 10 & 0.60 & 0.61 & 0.62 & 0.61 & 0.49 \\
\hline 15 & 1 & Wave 11 & 0.05 & 0.10 & 0.20 & 0.30 & 1.00 \\
\hline 15 & 1 & Wave 11 & 0.90 & 0.93 & 0.95 & 0.94 & 0.69 \\
\hline 15 & 1 & Waye 12 & 0.05 & 0.10 & 0.20 & 0.30 & 1.00 \\
\hline 15 & 1 & Wave 12 & 0.73 & 0.76 & 0.80 & 0.81 & 0.66 \\
\hline 15 & 2 & Waye 13 & 0.01 & 0.40 & 0.50 & 0.60 & 1.50 \\
\hline 15 & 2 & Wave 13 & 1.26 & 1.66 & 1.68 & 1.67 & 1.34 \\
\hline & & & 0.01 & 0.40 & 0.50 & 0.60 & 1.50 \\
\hline 15 & 2 & Wave 14 & 1.68 & 2.06 & 2.07 & 2.05 & 1.5 \\
\hline 15 & 2 & 15 & 0.01 & 0.40 & 0.50 & 0.60 & 1.50 \\
\hline 15 & 2 & e 15 & 2.14 & 2.59 & 2.52 & 2.45 & 1.77 \\
\hline 15 & 2 & & 0.01 & 0.40 & 0.50 & 0.60 & 1.50 \\
\hline 15 & 2 & e 16 & 1.82 & 2.39 & 2.41 & 2.40 & 1.88 \\
\hline
\end{tabular}

with duration of $1000 \mathrm{~s}$ were generated and sorted into four groups according to their statistical parameters. Each wave time series was simulated with the optimal resistance $R_{\mathrm{op}}$ and four other resistances $\left(R_{1}, R_{2}, R_{3}\right.$, and $\left.R_{4}\right)$ with values close to $R_{\mathrm{op}}$. Table II lists the wave state groups in which each wave time series presents the emulated resistances (first line) and their respective average captured powers (second line). Notice that $R_{2}$ and $R_{3}$ have a minimal variation with respect to the optimal resistance. It is verified that the maximum captured power is concentrated in $R_{2}, R_{\mathrm{op}}$, and $R_{3}$. The deviation of the captured power with respect to the optimal resistance does not exceed $3 \%$.

To examine the behavior of the $P_{\text {ratio }}-\gamma$ curves in the case of irregular waves, the wave time series which had more captured power for the optimal resistance in each wave state in Table II were selected. These time series were simulated with sampled values of emulated resistance and they had the $P_{\text {ratio }}-\gamma$ curve compared with their respective curve for regular wave obtained by the ideal case (Fig. 2). As shown in Fig. 11, the $P_{\text {ratio }}-\gamma$

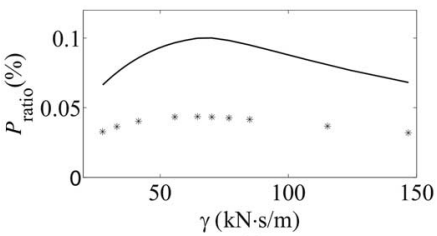

(a)

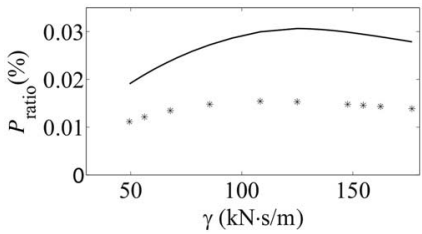

(c)

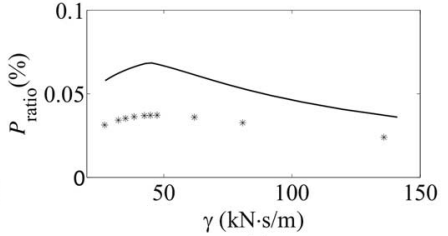

(b)

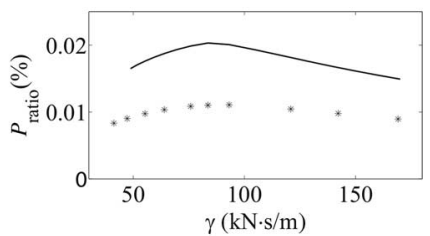

(d)
Fig. 11. Comparison of $P_{\text {ratio }}-\gamma$ curves between regular wave (solid line) and generated irregular wave (stars). (a) Wave 4. (b) Wave 6. (c) Wave 11. (d) Wave 15 .

TABLE III

MeAsured Wave Time SERIES INFORMATION

\begin{tabular}{cccccccc}
\hline \multirow{2}{*}{$\begin{array}{c}\text { Buoy } \\
\text { (station) }\end{array}$} & \multicolumn{2}{c}{ Location } & Date & Hour & $T_{e}$ & $H_{s}$ \\
\cline { 2 - 4 }$n$ & Latitude & Longitude & & & & & \\
\hline 043 & 33.219799 & -117.439400 & $2015-04-03$ & $13: 03$ & 9.25 & 1.0 \\
111 & 34.166916 & -119.434647 & $2015-04-02$ & $06: 43$ & 7.50 & 1.9 \\
043 & 33.219799 & -117.439400 & $2014-12-16$ & $06: 31$ & 14.75 & 1.1 \\
036 & 46.857719 & -124.243874 & $2014-03-23$ & $03: 46$ & 14.75 & 1.8 \\
\hline
\end{tabular}

curves in the simulations have a similar shape, in the sense that the captured power increases with the damping coefficient up to a maximum point, and it then starts to decrease. It can be noted that the $P_{\text {ratio }}-\gamma$ curves are quite different in $P_{\text {ratio }}$, because regular waves carry more energy per meter of wavefront, as shown in (16) and (17). However, for both regular and irregular waves, the optimal damping coefficients are almost coincident. Thus, it is possible to conclude that mapping the maximum power point for a combination of wave heights and periods for regular waves and applying the optimal values of the load seen by the electrical generator to irregular waves improves the efficiency of the system compared to any other electrical load.

\section{Simulations With Real Wave Data}

In this part, the system was simulated with four wave time series measured from real seas to validate the results obtained from the numerically generated wave time series in Section IV-B. For that, the measured data were collected from different buoys available in [45], where the displacement time series sampled at $1.28 \mathrm{~Hz}$, with sea states close to the presented in Table II, is provided. Information about the wave data is presented in Table III, where the buoy reference (station), the location in terms of latitude and longitude, the date and hour of measurement, and the significant wave height and energy period calculated according to [21] for the first $1000 \mathrm{~s}$ are indicated.

The methodology used to assess the results is the same as in Section IV-B, and Table IV presents the results in the same format as Table II. The average captured power for each wave time 
TABLE IV

COMPARISON OF THE CAPTURED POWER FOR IRREgular WAVE Profiles Measured From Real Seas With Different VALUES OF EMULATED RESISTANCE

\begin{tabular}{|c|c|c|c|c|c|c|c|}
\hline \multirow{4}{*}{$T_{e}(\mathrm{~s})$} & \multirow{4}{*}{$H_{s}(\mathrm{~m})$} & \multirow{4}{*}{$\begin{array}{l}\text { Wave time } \\
\text { series }\end{array}$} & \multicolumn{5}{|c|}{ Emulated resistances $(\Omega)$} \\
\hline & & & $R_{1}$ & $R_{2}$ & $R_{\mathrm{op}}$ & $R_{3}$ & $R_{4}$ \\
\hline & & & \multicolumn{5}{|c|}{ Average captured power $(\mathrm{kW})$} \\
\hline & & & $P_{R_{1}}$ & $P_{R_{2}}$ & $P_{R_{\mathrm{op}}}$ & $P_{R_{3}}$ & $P_{R_{4}}$ \\
\hline \multirow{2}{*}{9.25} & \multirow{2}{*}{1.0} & \multirow{2}{*}{ Wave 17} & 0.20 & 0.50 & 0.60 & 0.70 & 1.50 \\
\hline & & & 0.93 & 1.06 & 1.08 & 1.09 & 1.02 \\
\hline \multirow{2}{*}{7.50} & \multirow{2}{*}{1.9} & \multirow{2}{*}{ Wave 18} & 0.10 & 1.40 & 1.50 & 1.60 & 2.50 \\
\hline & & & 2.32 & 3.90 & 3.90 & 3.89 & 3.60 \\
\hline \multirow{2}{*}{14.75} & \multirow{2}{*}{1.1} & \multirow{2}{*}{ Wave 19} & 0.05 & 0.20 & 0.30 & 0.40 & 2.50 \\
\hline & & & 0.78 & 0.83 & 0.84 & 0.83 & 0.42 \\
\hline \multirow{2}{*}{14.75} & \multirow{2}{*}{1.8} & \multirow{2}{*}{ Wave 20} & 0.10 & 0.40 & 0.50 & 0.60 & 1.00 \\
\hline & & & 1.68 & 1.92 & 1.91 & 1.88 & 1.71 \\
\hline
\end{tabular}

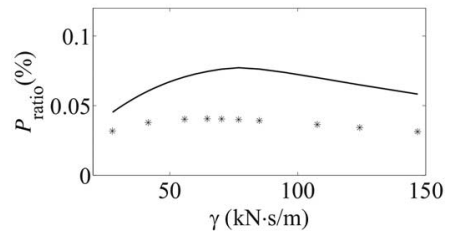

(a)

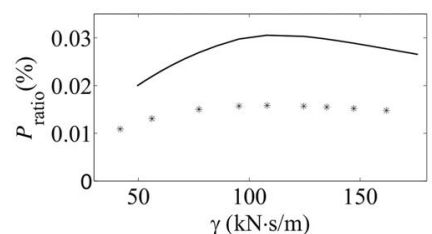

(c)

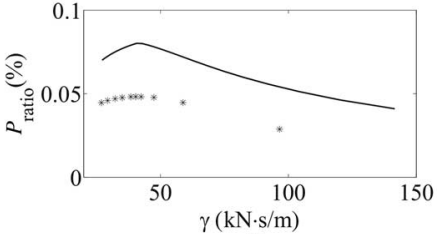

(b)

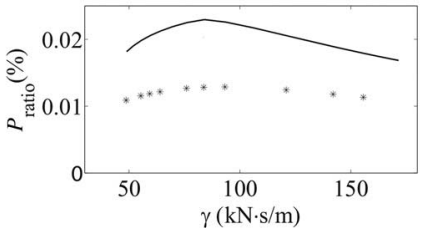

(d)
Fig. 12. Comparison of $P_{\text {ratio }}-\gamma$ curves between regular wave (solid line) and measured irregular wave from real seas (stars). (a) Wave 17. (b) Wave 18. (c) Wave 19. (d) Wave 20

series (waves 17, 18, 19, and 20) was obtained from the system set with five different emulated resistances, including the optimal resistance ( $R_{1}, R_{2}, R_{3}, R_{4}$, and $\left.R_{\mathrm{op}}\right)$. It can be seen that the maximum values of average captured power are concentrated around $R_{\mathrm{op}}$, as in the case of generated waves. The maximum deviation of the maximum average captured power with respect to the optimal resistance is $1 \%$, lower than the case of generated waves. Another analysis is the comparison of $P_{\text {ratio }}-\gamma$ curves of the measured wave time series and its respective regular wave as shown in Fig. 12, where the results are similar to those presented in Section IV-B. On the other hand, despite the fact that the sea states of the measured wave time series are slightly different from the generated wave time series, the $P_{\text {ratio }}-\gamma$ curves are very similar both in magnitude and in shape, showing the validity of the proposed strategy for real seas.

\section{CONCLUSION}

A good control strategy applied to the WEC is important to make its operation efficient. In fact, many control strategies have been developed for different types of WEC to maximize the captured power. The challenge in designing these types of controllers is to tune them by taking into account the nondeterministic characteristics of the waves. The proposed method, which is defined by mapping the optimal values for regular waves, proved effective when applied to real seas as long as the statistical parameters are identified. Simulations with different wave profiles have shown that the maximum captured power is around the optimal resistance previously identified, and that the $P_{\text {ratio }}-\gamma$ curves of an irregular wave and the ideal case have similar behavior. In addition, the implemented controller of the boost rectifier has shown to be a feasible way to control the electromagnetic force in this system. Although an efficiency drop is expected for large buoy motions, the work described in this paper shows that the proposed strategy works efficiently for height and period values where the higher order hydrodynamic effects can be neglected.

\section{REFERENCES}

[1] J. Falnes, "A review of wave-energy extraction," Mar. Struct., vol. 20, no. 4, pp. 185-201, 2007.

[2] B. Drew, A. Plummer, and M. N. Sahinkaya, "A review of wave energy converter technology," Proc. Inst. Mech. Eng. A. J. Power Energy, vol. 223 , no. 8, pp. 887-902, 2009.

[3] A. F. d. O. Falcão, "Wave energy utilization: A review of the technologies," Renew. Sustain. Energy Rev., vol. 14, no. 3, pp. 899-918, 2010.

[4] E. Lejerskog et al., "Lysekil research site, sweden: A status update," in Proc. 9th Eur. Wave Tidal Energy Conf., Southampton, U.K., 2011, pp. 1-7.

[5] L. Sjökvist, R. Krishna, M. Rahm, V. Castellucci, A. Hagnestål, and M. Leijon, "On the optimization of point absorber buoys," J. Mar. Sci. Eng., vol. 2, no. 2, pp. 477-492, 2014.

[6] J. Ringwood, G. Bacelli, and F. Fusco, "Energy-maximizing control of wave-energy converters: The development of control system technology to optimize their operation," IEEE Control Syst., vol. 34, no. 5, pp. 30-55, Oct. 2014

[7] P. Garcia-Rosa and J. Ringwood, "On the sensitivity of optimal wave energy device geometry to the energy maximizing control system," IEEE Trans. Sustain. Energy, to be published.

[8] A. Clément et al., "Wave energy in Europe: Current status and perspectives," Renew. Sustain. Energy Rev., vol. 6, no. 5, pp. 405-431, 2002.

[9] S. H. Salter, J. Taylor, and N. Caldwell, "Power conversion mechanisms for wave energy," Proc. Inst. Mech. Eng. M. J. Eng. Marit. Environ., vol. 216, no. 1, pp. 1-27, 2002.

[10] E. Tedeschi and M. Molinas, "Tunable control strategy for wave energy converters with limited power takeoff rating," IEEE Trans. Ind. Electron., vol. 59, no. 10, pp. 3838-3846, Oct. 2012.

[11] H. Yavuz, T. Stallard, A. McCabe, and G. A. Aggidis, "Time series analysis-based adaptive tuning techniques for a heaving wave energy converter in irregular seas," Proc. Inst. Mech. Eng. A. J. Power Energy, vol. 221, no. 1, pp. 77-90, 2007.

[12] F. Fusco and J. V. Ringwood, "Short-term wave forecasting for real-time control of wave energy converters," IEEE Trans. Sustain. Energy, vol. 1, no. 2, pp. 99-106, Jul. 2010.

[13] J. A. Cretel, G. Lightbody, G. P. Thomas, and A. W. Lewis, "Maximisation of energy capture by a wave-energy point absorber using model predictive control," in Proc. 18th IFAC World Congr., Milano, Italy, 2011, pp. 3714-3721.

[14] J. Hals, J. Falnes, and T. Moan, "Constrained optimal control of a heaving buoy wave-energy converter," J. Offshore Mech. Arct. Eng., vol. 133, no. 1, p. 011401, 2011.

[15] E. Abraham and E. C. Kerrigan, "Optimal active control and optimization of a wave energy converter," IEEE Trans. Sustain. Energy, vol. 4, no. 2, pp. 324-332, Apr. 2013.

[16] G. Bacelli, P. Balitsky, and J. V. Ringwood, "Coordinated control of arrays of wave energy devices-Benefits over independent control," IEEE Trans. Sustain. Energy, vol. 4, no. 4, pp. 1091-1099, Oct. 2013.

[17] M. Eriksson, R. Waters, O. Svensson, J. Isberg, and M. Leijon, "Wave power absorption: Experiments in open sea and simulation," J. Appl. Phys., vol. 102, no. 8, pp. 084 910-084 910, 2007. 
[18] C. Boström , B. Ekergard, R. Waters, M. Eriksson, and M. Leijon, "Linear generator connected to a resonance-rectifier circuit,” J. Ocean. Eng., vol. 38, pp. 255-262, 2013.

[19] C. Boström and M. Leijon, “Operation analysis of a wave energy converter under different load conditions,” IET Renew. Power Gener., vol. 5, no. 3, pp. 245-250, May 2011.

[20] S. Tyrberg, R. Waters, and M. Leijon, "Wave power absorption as a function of water level and wave height: Theory and experiment," IEEE J. Ocean. Eng., vol. 35, no. 3, pp. 558-564, Jul. 2010.

[21] M. E. McCormick, Ocean Engineering Mechanics With Applications, vol. 201. Cambridge, U.K.: Cambridge Univ. Press, 2010.

[22] H. O. Berteaux, Buoy Engineering. Hoboken, NJ, USA: Wiley, 1976.

[23] W. Cummins, "The impulse response function and ship motions," Schiffstechnik, vol. 9, pp. 101-109, 1962.

[24] Ansys Inc. (2015). ANSYS AQWA, Version 15.0, Canonsburg, PA, USA [Online]. Available: http://www.ansys.com/Products/Other+Products/ ANSYS+AQWA

[25] T. Perez, Ship Motion Control Course Keeping and Roll Stabilisation Using Rudder and Fins. New York, NY, USA: Springer, 2006.

[26] E. Kristiansen and O. Egeland, "Frequency-dependent added mass in models for controller design for wave motion damping," in Proc. 6th IFAC Conf. Manoeuvr. Control Mar. Craft (MCMC'03), Girona, Spain, Sep. 17-19, 2003.

[27] E. Jefferys, “Simulation of wave power devices,” Appl. Ocean Res., vol. 6, no. 1, pp. 31-39, 1984

[28] Z. Yu and J. Falnes, "State-space modelling of a vertical cylinder in heave,” Appl. Ocean Res., vol. 17, no. 5, pp. 265-275, 1995.

[29] T. Perez and T. I. Fossen, "Time-vs. frequency-domain identification of parametric radiation force models for marine structures at zero speed," Model. Ident. Control, vol. 29, no. 1, pp. 1-19, 2008.

[30] P. Kundur, Power System Stability and Control, N. J. Balu and M. G. Lauby, Eds. New York, NY, USA: McGraw-Hill, 1994.

[31] H. Mendonca and S. Martinez, "Modeling of a wave energy converter connected to a resistive load," in Proc. IEEE 4th Int. Youth Conf. Energy (IYCE), 2013, pp. 1-6.

[32] G. Duclos, A. Babarit, and A. H. Clément, "Optimizing the power take off of a wave energy converter with regard to the wave climate," J. Offshore Mech. Arct. Eng., vol. 128, no. 1, pp. 56-64, 2006.

[33] J. Falnes, Ocean waves and oscillating systems. Cambridge, U.K.: Cambridge Univ. Press, 2002.

[34] M. Eriksson, J. Isberg, and M. Leijon, "Hydrodynamic modelling of a direct drive wave energy converter,” Int. J. Eng. Sci., vol. 43, no. 17, pp. 1377-1387, 2005.

[35] K. Nielsen and C. Plum, "Point absorber-Numerical and experimental results," in Proc. 4th Eur. Wave Energy Conf., 2001.

[36] Z. Feng and E. C. Kerrigan, "Declutching control of wave energy converters using derivative-free optimization," in Proc. 19th World Congr. Int. Fed. Autom. Control, 2014, pp. 7647-7652.
[37] B. Teillant, J. Gilloteaux, and J. Ringwood, “Optimal damping profile for a heaving buoy wave energy converter,” in Proc. IFAC Conf. Control Appl. Mar. Syst. (CAMS), 2010, pp. 393-398.

[38] A. Babarit and A. Clément, "Optimal latching control of a wave energy device in regular and irregular waves,” Appl. Ocean Res., vol. 28, no. 2, pp. 77-91, 2006.

[39] A. Babarit, M. Guglielmi, and A. H. Clément, "Declutching control of a wave energy converter,” Ocean Eng., vol. 36, no. 12, pp. 1015-1024, 2009.

[40] J. K.-H. Shek, D. Macpherson, M. Mueller, and J. Xiang, "Reaction force control of a linear electrical generator for direct drive wave energy conversion,” IET Renew. Power Gener., vol. 1, no. 1, pp. 17-24, Mar. 2007.

[41] J. A. Oskamp and H. T. Özkan-Haller, "Power calculations for a passively tuned point absorber wave energy converter on the oregon coast," Renew. Energy, vol. 45, pp. 72-77, 2012.

[42] R. W. Erickson and D. Maksimovic, Fundamentals of Power Electronics. New York, NY, USA: Springer, 2001.

[43] D. M. Brod and D. Novotny, "Current control of VSI-PWM inverters," IEEE Trans. Ind. Appl., vol. IA-21, no. 3, pp. 562-570, May 1985.

[44] The Specialist Committee on Waves (chaired by C. T. Stansberg), "Final report and recommendations to the 23rd ITTC,” in Proc. 23rd Int. Towing Tank Conf., vol. II, 2002, pp. 505-551.

[45] The Coastal Data Information Program. (2015). CDIP Historic Data [Online]. Available: https://cdip.ucsd.edu/ 